\title{
Custom-Made Bifurcated Prosthetic Graft for Aortoiliac Aneurysm Repair
}

\author{
Spyros Papadoulas, MD, PhD ${ }^{1}$ Stavros K. Kakkos, MD, MSc, PhD, DIC, RVT ${ }^{1}$ \\ loannis Ntouvas, MD, $\mathrm{PhD}^{1}$ Konstantinos Nikolakopoulos, MD, $\mathrm{PhD}^{1}{ }^{1}$ Polyzois Tsantrizos, MD, MSc ${ }^{1}$ \\ Chrysanthi Papageorgopoulou, MD, $\mathrm{MSc}^{10}$ Natasa Kouri, MD ${ }^{1}$ (1) \\ ${ }^{1}$ Department of Vascular Surgery, University Hospital of Patras, \\ Patras, Greece \\ Aorta (Stamford) 2021;9:88-91 \\ Address for correspondence Spyros Papadoulas, MD, PhD, \\ Department of Vascular Surgery, University Hospital of Patras, Rio, \\ Patras 26504, Greece (e-mail: spyros.papadoulas@gmail.com).
}

\author{
Abstract \\ Keywords \\ - abdominal aortic \\ aneurysm \\ - iliac aneurysm \\ - internal iliac \\ revascularization \\ - open repair \\ - technique
}

Revascularization of the internal iliac artery during open repair of aortoiliac aneurysms can be challenging, especially if there is a significant distance between the orifices of the internal and external iliac arteries owing to common iliac aneurysmal dilatation. We describe a technique involving insertion of an 18-mm tube graft between the proximal aortic neck and aneurysmal common iliac artery bifurcation. Revascularization of the contralateral external iliac artery is accomplished through an 8-mm side arm graft.

\section{Introduction}

Despite the evolution of endovascular techniques, repair of aortoiliac aneurysms is still performed by open surgery in a significant number of patients, particularly those with prolonged expected survival. Significant aneurysmal degeneration of the distal common iliac artery may lead to displacement of the orifices of the external and internal iliac arteries (EIA and IIA, respectively), making a formal end-to-end anastomosis with the graft limb of a bifurcated graft unsafe or even impossible. Several modifications of endovascular and open repair techniques have been described in the literature devised to overcome this problem. We describe an additional modification of the open reconstruction.

\section{Technique and Results}

A 79-year-old male with lower abdominal pain radiating to the lumbar spine was admitted to the hospital and underwent a computed tomography angiography that revealed an abdominal aortic aneurysm (AAA) measuring $8 \mathrm{~cm}$ in diameter and

received

December 20, 2019

accepted after revision

November 11, 2020

published online

October 7, 2021 extending to both EIA and IIA. The infrarenal neck measured $2.8 \mathrm{~cm}$ in diameter. Right and left common iliac aneurysms measured 4.2 and $3.8 \mathrm{~cm}$ in diameter, respectively (-Fig. 1). The right common iliac bifurcation measured $2.7 \mathrm{~cm}$ in diameter, while the distance between the origins of the EIA and IIA was $0.5 \mathrm{~cm}$. Past medical history was significant for coronary artery disease, a $4-\mathrm{cm}$ synchronous isolated descending thoracic aorta aneurysm, stroke without residual neurological deficit, and left inguinal hernia. During laparotomy, an intact aneurysm was found that was repaired with the technique described hereafter.

The patient was operated under general orotracheal anesthesia in the supine position. Through a standard midline laparotomy, the aortic neck and the iliac bifurcations were dissected out in the usual fashion. The AAA was opened longitudinally and an 18-mm aortic tube graft (PFTE, Goretex Vascular Graft, W. L. Gore and Associates Inc., Flagstaff, AZ) was interposed. Proximally, the graft was sutured in an endto-end fashion with the infrarenal aortic neck, and it was anastomosed in an end-to-end fashion with the right iliac bifurcation distally, encompassing both the orifices of the

\section{(C) 2021. The Author(s)}

This is an open access article published by Thieme under the terms of the Creative Commons Attribution License, permitting unrestricted use, distribution, and reproduction so long as the original work is properly cited. (https://creativecommons.org/licenses/by/4.0/)

Thieme Medical Publishers, Inc., 333 Seventh Avenue, 18th Floor, New York, NY 10001, USA 


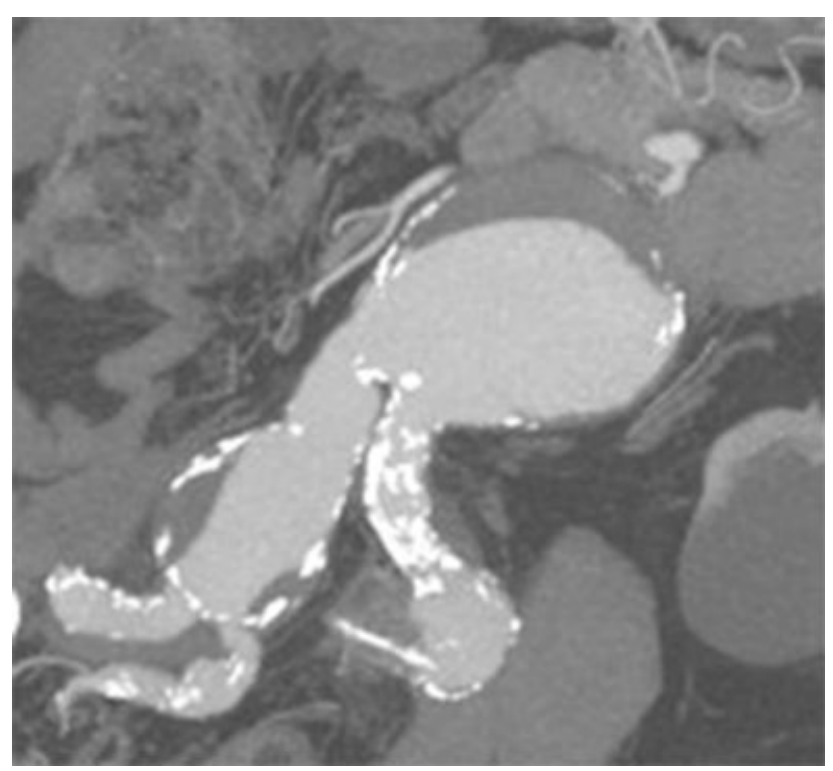

Fig. 1 Computed tomography angiography showing an abdominal aortic aneurysm of $8 \mathrm{~cm}$ in diameter extending in both iliac arteries.

right IIA and right EIA. A separate side arm/tube graft ( $8 \mathrm{~mm}$, Goretex Vascular Graft, W. L. Gore and Associates Inc.) was then anastomosed proximally with the aortic graft (in endto-side fashion) and distally with the left EIA (in end-to-end fashion), while the left IIA was ligated (-Figs. 2 and $\mathbf{3}$ ). Postoperative course was uneventful, and the patient left the hospital on the seventh postoperative day. He did not develop buttock claudication on the left side as a result of IIA ligation; he passed away 3 years later after a mechanical fall in his home and severe depression, which left him bedridden for about a month.

\section{Discussion}

Open repair of an AAA is considered complex in cases of concomitant common iliac and/or IIAs, especially if they are bilateral and involve the iliac bifurcation, requiring revascularization of at least one IIA. Approximately, 10 to $20 \%$ of AAAs are combined with iliac aneurysms. Isolated iliac aneurysm accounts for 2 to $7 \%$ and isolated IIA aneurysms for 0.3 to $0.5 \%$ of all abdominal aneurysms. ${ }^{1,2}$

An important guideline is the revascularization of at least one IIA, while the other one may be sacrificed. However, complications, such as buttock ischemia (including buttock claudication in 10-30\%), erectile dysfunction, and bowel and spinal cord ischemia, may rarely develop, and are more frequent after bilateral IIA ligation. ${ }^{3}$ Therefore, restoration of blood flow in one or both IIAs during repair of aortoiliac aneurysms or bilateral isolated iliac aneurysms is always desirable.

In some cases, a formal distal end-to-end anastomosis of the graft limb with the common iliac bifurcation is impossible due to long distance between the orifices of the IIA and EIA, owing to aneurysmal dilatation of the distal common iliac artery (-Fig. 4). If this pathology is bilateral, there is need for

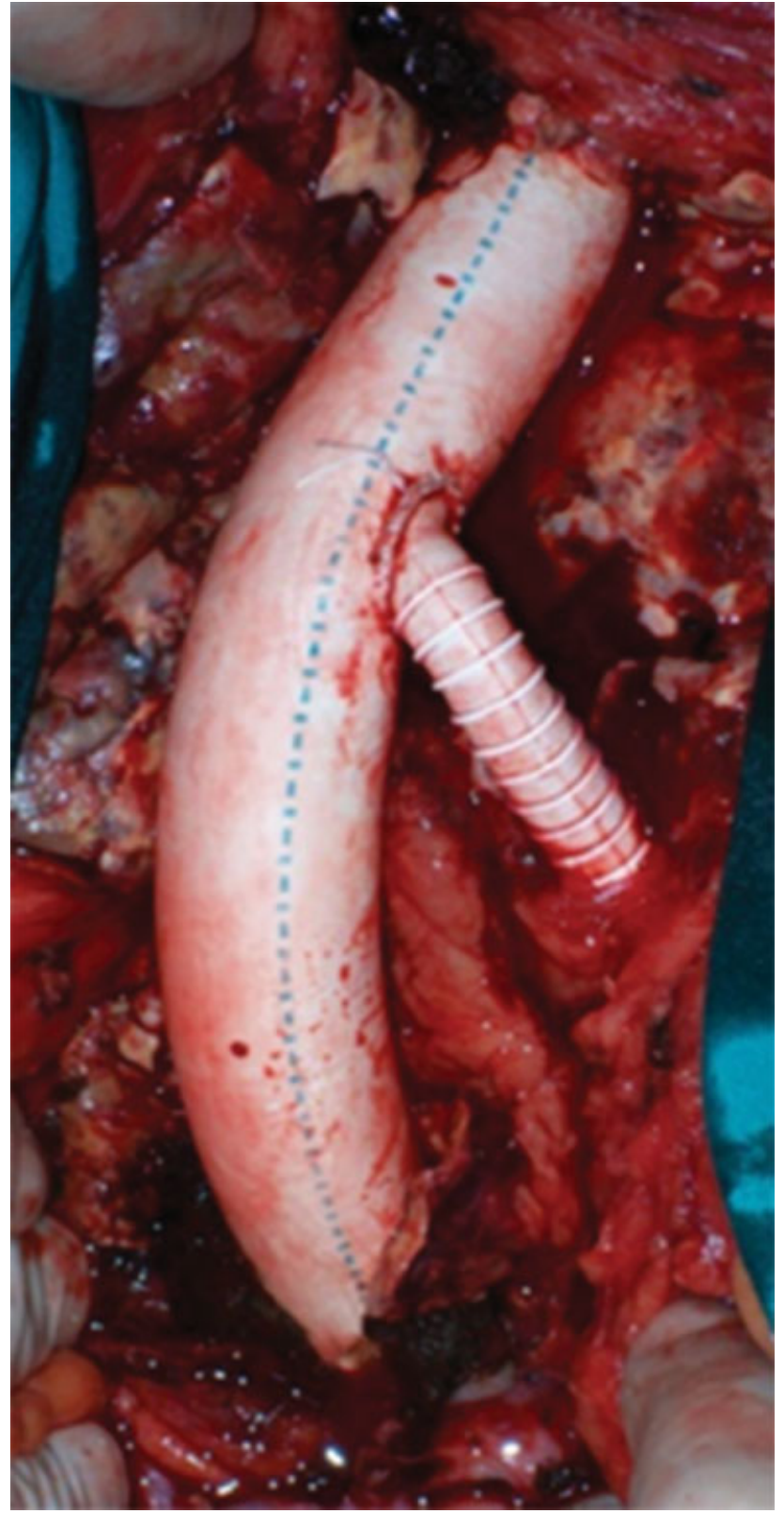

Fig. 2 Intraoperative photo after the reconstruction.

reimplantation of the IIA in the graft limb (end-to-side fashion), or revascularization using a jump graft, after the completion of the end-to-end anastomosis of the graft limb with the EIA, at least on one side (the contralateral IIA can be ligated). ${ }^{4}$ In comparison with this formal technique, the technique we describe herein may be performed more easily, it demands fewer anastomoses (four vs. five in case of repair with an aortoiliac graft anastomosed with the EIAs and revascularization of one IIA with a jump graft) and offers an alternative approach in emergency cases, if there is lack of the appropriate bifurcated graft due to logistic reasons.

Upon review of the literature, we found some alternative techniques that have been performed at least to one side (with contralateral IIA ligation) as follows:

- End-to-end anastomosis of the graft limb with the IIA end reimplantation of the EIA in the graft limb. ${ }^{5-7}$ 


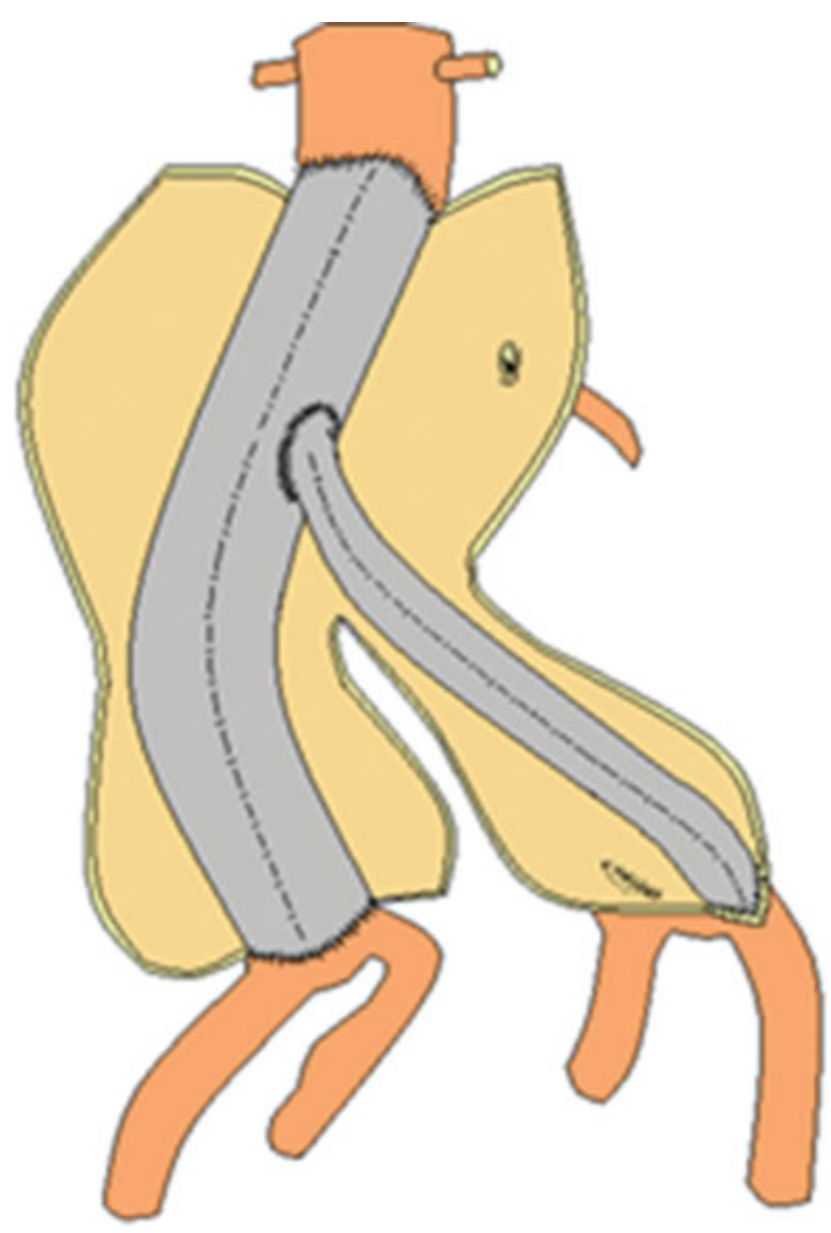

Fig. 3 A drawing depicting the reconstruction.

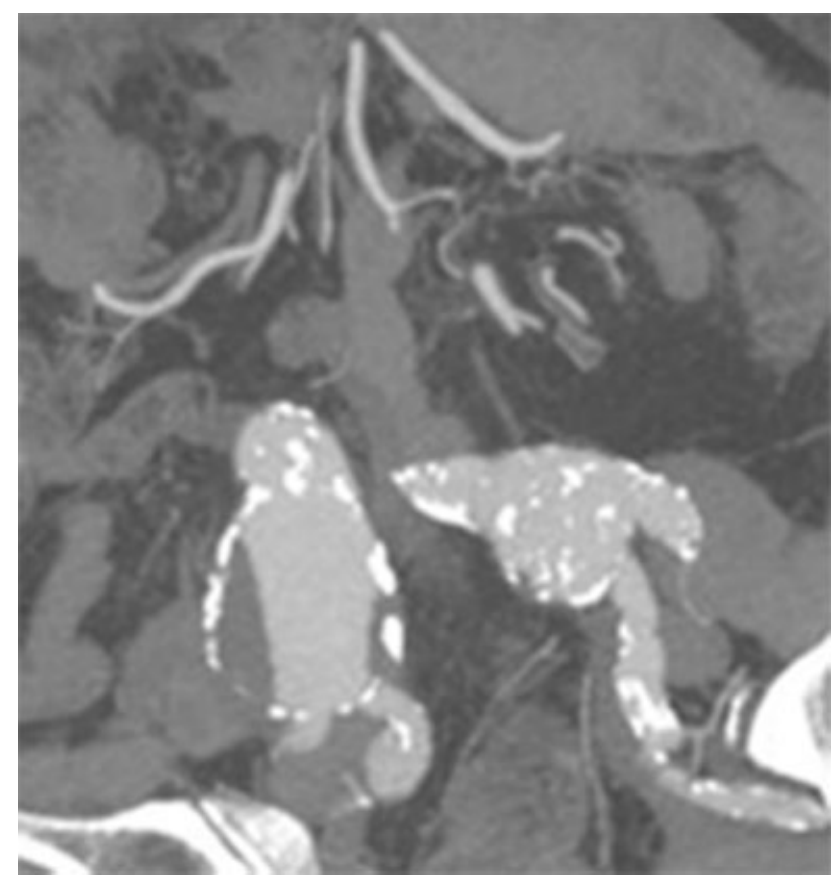

Fig. 4 Computed tomography angiography showing the remarkable distance between the orifices of the internal iliac artery and external iliac artery in both sides.
- Custom-made trifurcated graft which is tailored on table and provides a separate graft limb for revascularization of the IIA. ${ }^{6}$

- A specific tube graft with a nitinol stent at one end (Gore hybrid vascular graft, GVHG; W. L. Gore and Associates Inc., Flagstaff, AZ). It is anastomosed centrally with the graft limb of a bifurcated graft, and is fixed after deployment of its stent-portion in the IIA distally. ${ }^{1}$ It can be deployed over a 5-Fr Pruit catheter (LeMaitre Vascular, Burlington, MA) which is used to obtain distal control. This is important in cases of concomitant IIA aneurysms where the distal anastomosis may be time consuming and troublesome.

- The first AAA resection by Dubost et $\mathrm{al}^{8}$ was performed in an AAA without iliac bifurcation involvement treated with interposition of a tube allograft between the aortic neck and the right common iliac artery. The contralateral iliac axis was dissected free and the thrombosed IIA was transected and ligated to bring up the endarterectomized common iliac artery for an end-to-side anastomosis with the tube graft. This approach could be followed in case of wide distance between the iliac orifices ipsilaterally, by insertion of a prosthetic tube graft from the aortic neck down to the iliac bifurcation. Then it is anastomosed in the same way with the contralateral iliac artery.

\section{Conclusion}

In conclusion, depending on the aneurysm morphology and the difficulties arising in a specific operation, the surgeon decides if the modification, we describe here, can help to carry out the arterial reconstruction more easily.

\section{Funding}

None.

\section{Conflict of Interest}

The authors declare no conflict of interest related to this article.

\section{Acknowledgment}

None.

\section{References}

1 Tyagi S, Pineda D, Zheng H, Dougherty M, Calligaro K, Troutman D. A novel method for the treatment of bilateral hypogastric aneurysms using hybrid polytetrafluoroethylene graft. Vasc Endovascular Surg 2017;51(04):199-202

2 Kim JY, Kim DH, Quan C, et al. Treatment options for isolated iliac artery aneurysms and their impact on aortic diameter after treatment. Ann Surg Treat Res 2019;96(03):146-151

3 Bosanquet DC, Wilcox C, Whitehurst L, Cox A, Williams IM, Twine CPBritish Society of Endovascular therapy (BSET) Systematic review and meta-analysis of the effect of internal iliac artery exclusion for patients undergoing EVAR. Eur J Vasc Endovasc Surg 2017;53(04):534-548

4 Milite D, Campanile F, Tosato F, Pilon F, Zaramella M. Hypogastric artery bypass in open repair of abdominal aortoiliac aneurysm: a safe procedure. Interact Cardiovasc Thorac Surg 2010;10(05): 749-752 
5 Dorigo W, Pulli R, Troisi N, et al. The treatment of isolated iliac artery aneurysm in patients with non-aneurysmal aorta. Eur J Vasc Endovasc Surg 2008;35(05):585-589

6 Donas KP, Torsello G, Pitoulias GA, Austermann M, Papadimitriou DK. Surgical versus endovascular repair by iliac branch device of aneurysms involving the iliac bifurcation. J Vasc Surg 2011;53 (05):1223-1229
7 Patel NV, Long GW, Cheema ZF, Rimar K, Brown OW, Shanley CJ. Open vs. endovascular repair of isolated iliac artery aneurysms: A 12-year experience. J Vasc Surg 2009;49(05):1147-1153

8 Dubost C, Allary M, Oeconomos N. Resection of an aneurysm of the abdominal aorta: reestablishment of the continuity by a preserved human arterial graft, with result after five months. AMA Arch Surg 1952;64(03):405-408 